RESEÑA

DOI: $10.24844 / \mathrm{EM} 3203.10$

\title{
Enseñar a enseñar matemáticas. Un recorrido de estudio de investigación para la formación de profesores, de Maricela Soto y Luis Manuel Aguayo
}

\author{
Alejandra Avalos-Rogel ${ }^{1}$
}

\section{INTRODUCCIÓN}

La formación inicial de docentes de matemáticas es una línea de investigación que ha sido poco abordada por su complejidad, pues convergen prácticas, actores, saberes matemáticos y didácticos con diferentes estatus epistémicos, instituciones y culturas. En este tejido cósmico que no siempre es inteligible, Enseñar a enseñar matemáticas. Un recorrido de estudio de investigación para la formación de profesores de Marcela Soto y Luis Manuel Aguayo ofrece ordenamiento y comprensión de este proceso complejo. Ese trabajo se presenta como el reporte de una investigación sobre el diseño y puesta en marcha de un dispositivo de formación inicial de futuros docentes de la licenciatura en Educación Primaria en relación a la proporcionalidad, fundamentado en la Teoría Antropológica de lo Didáctico (TAD).

Responder a la pregunta "¿Qué efectos tienen sobre la formación de los estudiantes ciertas tareas matemáticas y didácticas especialmente diseñadas para tal propósito?" brindó a los autores la posibilidad de abordar las siguientes configuraciones conceptuales: las características de la formación inicial de docentes de matemáticas, la proporcionalidad como un texto del saber en el

\footnotetext{
${ }^{1}$ Escuela Normal Superior de México, alejandraavalosrogel@gmail.com, orcid.org/0000-0003-3650-4887
} 
tránsito de la aritmética al álgebra, los Recorridos de estudio e investigación, que son los dispositivos que se han diseñado desde la TAD para la construcción de praxeologías matemáticas, las etapas formativas de los estudiantes para maestros en la etapa inicial, y sobre los tejidos conceptuales de la TAD con la Teoría de las Situaciones Didácticas para analizar las prácticas docentes de los estudiantes en formación.

Para esta investigación los autores hacen el seguimiento durante un semestre de un grupo de estudiantes normalistas en formación de la licenciatura en Educación Primaria de los últimos semestres, en el estado de Zacatecas, en México. Dos de esos estudiantes llevaron sus diseños de propuestas de enseñanza al aula, con estudiantes de primaria.

En esta reseña se recuperan algunas ideas de esos aspectos, con el ánimo de invitar a la lectura del libro.

\section{LA FORMACIÓN INICIAL DE DOCENTES DE MATEMÁTICAS DESDE LA TAD}

Los autores distinguen dos perspectivas sobre la relación entre didáctica y formación de profesores: la cognitiva o estudios sobre el pensamiento del profesor, y la epistemológica o estudios sobre los saberes (praxeologías). La investigación se inscribe en la segunda, pues se presenta una reflexión sobre la actividad humana y el tipo de saberes involucrados en ella. En particular recuperan la categoría de organizaciones praxeológicas o praxeologías y sus cuatro componentes: las tareas a resolver, las técnicas que las resuelven, la tecnología o discurso que explica y justifica la técnica, y la teoría que justifica la tecnología.

Se parte de los hallazgos de la TAD de que una Organización Matemática gira en torno a un objeto matemático y una Organización Didáctica considera el estudio de la praxeología matemática. Se afirma que los docentes recuperan y entrelazan ambas en su actividad profesional, porque los dos tipos de praxeología siempre están codeterminados: una praxeología didáctica no puede elaborarse ni difundirse sin una praxeología matemática y viceversa.

En este momento el texto presenta una cuestión crucial. En el sistema de formación ¿̇uál es la naturaleza del objeto a estudiar? Porque en el caso de la formación de docentes no se trata de saberes, sino de recuperar las invariantes de las prácticas humanas, o praxeologías, que se realizan en determinadas instituciones. 
Primero, es necesario entender que hay seis momentos didácticos, no cronológicos, que modelizan todo proceso de estudio: el momento del primer encuentro (o reencuentro) con la organización $\mathrm{O}$ que está en juego, y que puede presentarse como un problema o una pregunta; el momento de la exploración del tipo de tareas Ty de la técnica ci relativa a ellas; el momento de la constitución del entorno tecnológico-teórico relativo a $\tau i$; el trabajo de técnica, volviéndola más eficaz; el momento de la institucionalización, que tiene por objeto precisar la $\mathrm{OM}$; y el momento de la evaluación, para verificar lo aprendido.

Posteriormente hay que considerar los procesos normalizadores que tienen lugar en el sistema escolar, en las escuelas formadoras como las escuelas normales, las huellas de los maestros innovadores y las normas que se derivan de la investigación en didáctica, y que se convierten en restricciones y determinantes institucionales.

Finalmente los autores recuperan de Chevallard (1994) el sistema de tareas del profesor, mismo que se organiza en dos categorías:

- tareas relativas a la concepción, organización y gestión de dispositivos de estudio (determinar las praxeologías matemáticas escolares)

- tareas de ayuda al estudio y de dirección de estudio y de enseñanza.

Para el caso de la formación docente, los autores apuestan por aproximaciones a la actividad matemática y su estudio, a la actividad didáctica y su estudio y a una orientación o perspectiva desde la TAD que las vincula con la formación de profesores, al ser un soporte teórico para analizarla.

\section{LA EVOLUCIÓN PRAXEOLÓGICA DE LA PROPORCIONALIDAD}

No es posible proponer una Organización Didáctica sin la Organización Matemática de referencia. Y para ello se requiere describir el Modelo epistemológico de referencia (MER), con el fin de reconocer la naturaleza del objeto matemático y los fenómenos matemáticos y didácticos que produce. En este libro se analiza la proporcionalidad como un MER. El argumento de los autores se centra en identificar un recorrido epistémico de la organización clásica a la organización funcional de la proporcionalidad, recorrido que también estará en la base del dispositivo propuesto para la formación de los futuros docentes. 
Los autores recuperan algunos elementos de la organización clásica estudiados por otros didactas. Por ejemplo la razón con sus diversas acepciones y significados, entre los que se encuentra la comparación entre cantidades de la misma magnitud; la cuantificación de una relación multiplicativa como fracción parte-todo, cociente, operador y razón; la comparación de objetos heterogéneos y no relacionados con la fracción; como división indicada; y finalmente como relación de equivalencia.

El segundo elemento es la proporción, sus propiedades y significados como el de igualdad y el de equivalencia de dos razones geométricas; la propiedad fundamental de las proporciones $(a / b=c / d$ si y sólo si $a d=b c)$; y la proporción discreta y proporción continua.

La relación directamente proporcional de magnitudes se muestra como uno de los grandes temas en este MER, en particular las diversas caracterizaciones para presentar la relación directamente proporcional de magnitudes, explícitamente e implícitamente relacionadas por una función $\mathrm{f}(\mathrm{x})$. La importancia de este análisis también radica en la identificación de tareas: tareas de valor faltante, tareas de comparación; y por el tipo de técnicas en la Organización clásica: la regla de tres y la reducción a la unidad.

Los autores recuperan los estudios donde se analizan los contextos donde se utilizan las proporciones, y señalan la diferencia entre proporción y el razonamiento proporcional y la algebrización de la proporcionalidad en diversos niveles.

\section{EL ANÁLISIS ECOLÓGICO DE LA INSTITUCIÓN}

Lo realizan considerando desde la TAD que las instituciones educativas tienen diversos mecanismos para establecer las normas que van a conformar OM y OD. Uno de ellos es el curriculum. En el caso de las matemáticas, los contenidos y los enfoques metodológicos han ido a la par con el avance de la investigación en Educación Matemática. Un ejemplo, es el enfoque de la resolución de problemas. Otro es el abordaje de la proporcionalidad que también se ha dado a la par con los avances de la investigación.

También analizan cómo se presenta la proporcionalidad en los programas de estudio de la educación básica y en la escuela normal y cómo esto determina las prácticas de los docentes y de los estudiantes normalistas. Una perspectiva es la Modelación matemática, interpretada desde el modelo epistemológico subyacente, desde las culturas institucionales y desde la noosfera, lugar 
simbólico donde se da la interacción del sistema de enseñanza con el entorno social. Se trata de una dimensión económica, entendida como la coordinación de componentes de la relación institucional con la actividad matemática, y que Chevallard (2002) había caracterizado como niveles de codeterminación matemático didácticos.

Muestran cómo desde el trabajo en torno a la proporción en la primaria se prevé una articulación con la función lineal de la escuela secundaria, y en el caso del curriculum de la educación normal, en el curso Aritmética: su enseñanza y su aprendizaje, la proporción se presenta como una delimitación de saberes parciales o desincretización del saber (Chevallard. 1991) en cuatro temas: conceptos de razón y proporción a través de situaciones, el porcentaje y su representación gráfica, el cálculo de porcentajes y la variación proporcional directa.

Una observación interesante de los autores es que el "texto del saber" en este curso se presenta a estudiantes normalistas de acuerdo a la manera como sería leído, esto es, los estudiantes asumen diversas funciones lectoras: como profesor "analista" de situaciones; como "aprendiz" de matemáticas; como estudiante y profesional reflexivo de sus propios procedimientos y de los posibles procedimientos de sus alumnos en la razón y proporción (praxeología didáctica); como estudiante que realiza un esfuerzo metacognitivo para entender los procedimientos y las técnicas en tareas matemáticas que involucran el porcentaje o la variación proporcional.

En el análisis del segundo curso del programa de estudios, denominado Algebra: su aprendizaje y enseñanza, los autores advierten que el trabajo con la proporcionalidad, el análisis de patrones numéricos y de representaciones tabulares, gráficas y analíticas, llevan a los estudiantes a profundizar en el concepto de función lineal, y reconocerla como una herramienta eficaz para la modelización.

Consideran que en este curso hay tareas relacionadas con la Organización didáctica: la revisión y análisis de contenidos de matemáticas de primaria, y en menor medida, el análisis de propuestas didácticas, el diseño de secuencias didácticas para la educación primaria, y el diseño de problemas que requieren del uso del álgebra. Un hallazgo sorprendente de los autores al analizar la cantidad de tareas para cada tema es que ino hay ninguna actividad didáctica para la proporcionalidad y más de la mitad está destinada a la enseñanza del porcentaje! 


\section{LOS RECORRIDOS DE ESTUDIO E INVESTIGACIÓN PARA LA FORMACIÓN DE PROFESORES (REI-FP)}

Después de abordar la idea de cómo un instrumento para la actividad matemática modifica la naturaleza de las organizaciones matemático didácticas, los autores presentan un Recorrido de Estudio de Investigación para la formación de docentes (REI-FP) como un dispositivo. En un REl se parte de una pregunta generatriz $(Q 0)$ que se desglosa en cuestiones de diferente grado, para el estudio de un saber específico en donde se trastocan nociones relacionadas entre sí, y que requieren de búsquedas para acceder a información que posibilite la construcción de respuestas.

Los autores proponen un REI-FP, cuya pregunta generatriz Q0 fue la siguiente: ¿Cuáles son las praxeologías matemáticas y didácticas ligadas a la proporcionalidad a incluirse en la formación de profesores?

El REl-FP estuvo organizado en 4 módulos de acuerdo a los diferentes roles que un estudiante puede adoptar en la construcción de una organización matemática entrelazada con una organización didáctica.

Primer módulo. M1: "Vivir un REl". Rol "Aprendiz de matemáticas"

Segundo módulo. M2: "Analizar el REl". Rol "Profesor analista"

Tercer módulo. M3: "Diseñar un REl". Rol "Ingeniero diseñador"

Cuarto módulo. M4: "Gestionar y experimentar un REl". Rol "Profesor en acto"

El primer módulo M1: "Vivir un REl", los estudiantes en su calidad de aprendices de matemáticas abordaron una situación de proporcionalidad denominada "El petróleo", sobre los tipos y costos del crudo en México, y que sirvió como base y referente para desarrollar el resto de los módulos.

La pregunta generatriz del módulo fue la siguiente:

Q1: ¿Qué técnicas $\tau$ y discursos tecnológicos teóricos $(\theta, \Theta)$ despliegan los profesores en formación para resolver tareas $(t)$ matemáticas en la reconstrucción de una praxeología ligada la proporcionalidad. (Aguayo y Soto, 126).

Las tareas planteadas fueron las siguientes: 1 . Tarea de valor faltante y magnitud variable, con magnitudes enteras, fraccionarias, decimales, con valor faltante y magnitud entera; 2. Tarea de la representación gráfica de la variación proporcional, y praxeologías para los valores "y" de los puntos; 3. Tarea con tablas de variación proporcional, costos menores al valor unitario; 4. Tarea de variación 
proporcional de tres magnitudes; 5 . Tarea de variación proporcional compuesta y la búsqueda del modelo algebraico. Esta última tarea se apoyó en una indagación con "especialistas", o en textos matemáticos, con la finalidad de adquirir una técnica para resolver la tarea. Este aspecto acentuaba el carácter de recorrido y de investigación del REl.

En este módulo del REI-FP los estudiantes normalistas consolidaron un modelo algebraico y desarrollaron técnicas algebraicas que fueron justificadas y validadas.

En el segundo módulo. M2: "Analizar el REl", los estudiantes normalistas en su rol de "Profesores analistas" abordan tareas relativas al análisis de una praxeología didáctica, lo que implica reconocer los alcances y restricciones que una institución ha establecido para el estudio de un saber.

La pregunta generatriz del módulo fue la siguiente:

Q2: ¿Qué discurso tecnológico-teórico $(\theta, \Theta)$ despliegan los profesores en formación durante el desarrollo de tareas $(t)$ referidas al análisis de una praxeología didáctico-matemática ligada a la proporcionalidad. (Aguayo y Soto, 180)

El propósito es entonces identificar el discurso tecnológico de los profesores en formación al reflexionar sobre los conceptos, tareas y técnicas de la proporcionalidad en el REI-FP que vivieron.

En una dimensión descriptiva, los autores recuperan mediante una entrevista semiestructurada las tareas que resolvieron y las técnicas que utilizaron.

En una dimensión predictiva, cuando se invita a los estudiantes a asumir el rol de profesor, reflexionan sobre las elecciones locales en función de las características de una tarea. De ahí que sea posible vislumbrar, como docente, sus posibilidades de acción, decisión, control y validación a trabajar de modo independiente al formador o sea se prevén los posibles comportamientos.

En el tercer módulo. M3: "Diseñar un REl", los estudiantes asumen el rol de "Ingeniero diseñador", y se ubican en la dimensión matemática para seleccionar problemas que constituyan un REl, esto es, propiciar ciertas tareas que provoquen el uso de una o varias técnicas y un discurso tecnológico con el cual las justifican y validan.

Q3: ¿Cómo organizan y diseñan los profesores en formación el estudio de la proporcionalidad a través de una organización didáctica? (Aguayo y Soto, 219). 
La elección está condicionada por restricciones institucionales y por un Marco Epistemológico de Referencia, y está fundamentada en un Modelo Didáctico de Referencia, que en este caso fue la Teoría de las Situaciones Didácticas. Los estudiantes normalistas diseñaron dos REI dirigidos a niños de sexto año de primaria muy similares a los que habían vivido en la situación de aprendices. Estos recorridos incluyen cuatro tareas cuya solución implica la utilización de técnicas como el valor unitario, el modelo tabular, la comparación de razones y el uso de la proporcionalidad con tres magnitudes con un rango numérico de dos cifras. En relación a la praxeología didáctica, la planeación tenía la misma estructura de un estudio: el primer encuentro, la constitución del entorno tecnológico-teórico relativo a la técnica, y eventualmente, la inclusión de la institucionalización, aspecto que fue difícil contemplar. Lo destacable es que no incluyen momentos para el trabajo de la técnica, ni de evaluación de la praxeología, que resultan determinantes en la ejecución de un REI.

En el cuarto módulo M4: "Gestionar y experimentar un REl". Rol "Profesor en acto", los estudiantes en su calidad de docentes frente a grupo con alumnos reales, condujeron los procesos de estudio no lineales, de la proporcionalidad en la escuela primaria.

La pregunta generatriz del módulo fue la siguiente:

Q4: ¿Qué tareas $(t)$, técnicas $(\tau)$ y discurso tecnológico-teórico $(\theta, \Theta)$ utilizan los profesores en formación para gestionar el proceso de estudio de una praxeología matemática ligada la proporcionalidad. (Aguayo y Soto, 237)

En el marco de la investigación, esto supone algunas consideraciones para el estudiante normalista:

- Una comprensión de la praxeología matemática de la proporcionalidad que reconstruyó en el primer módulo de su recorrido.

- Claridad sobre las praxeologías didácticas adecuadas a un estudio, la existencia de saberes matemáticos construidos gracias al proceso de estudio y la manera específica en el que el proceso de estudio ha de desarrollarse.

Los autores analizaron la experimentación que hicieron los estudiantes normalistas de sus Recorridos de Estudio e Investigación con un grupo de alumnos de primaria. El objetivo del Análisis consistió en identificar las tareas didácticas que 
ponen en juego, las técnicas que implementan para gestionar dichas tareas y los elementos tecnológicos-teóricos que las justifican.

Un señalamiento que hacen los autores es que la experimentación de los REl fue una tarea extracurricular por las restricciones curriculares de los tiempos de clase, particularmente las lecciones del libro de texto y los temas que debían abordarse. Sin embargo, el tema era familiar para los alumnos, porque ya contaban con un referente praxeológico inicial adquirido en la solución de tareas de proporcionalidad en grados anteriores.

Para el análisis de los episodios de la experimentación de los REl, se estableció la siguiente estructura praxeológica mínima de los recorridos de estudio, lo que permitió a los autores hacer el seguimiento y la evaluación de la implementación.

A. Un problema didáctico matemático al que el sistema de enseñanza tiene que dar respuesta

B. Una institución concreta donde se plantea el problema en cuestión

C. La razón de ser de las organizaciones matemáticas

- Resolver tareas matemáticas

- Identificar las técnicas que se utilizan para resolver tareas

- Identificar la evolución de las técnicas

- Comprender el funcionamiento y significado de modelos algebraicos en torno a la proporcionalidad

D. Elegir una cuestión o situación generatriz

E. Un dispositivo didáctico que puede ser considerado como un proceso de ingeniería matemática denominado organizaciones matemáticas locales relativamente completas

F. Un contrato didáctico donde se observarán las responsabilidades del profesor y del alumno ante las situaciones de aprendizaje y enseñanza

G. Una organización matemática local relativamente completa en la que se genera el proceso de estudio de la actividad matemática

Y el análisis del planteamiento de los siguientes tipos de tareas de proporcionalidad:

T1. Tareas de valor faltante que se resuelven con la técnica del valor unitario.

T2. Tareas ligadas al valor faltante en Tablas de variación proporcional que se resuelven con la regla de tres. 
T3. Tareas vinculadas con el uso de la razón que requieren la comparación de razones como técnica.

T4. Tareas asociadas con la variación proporcional de tres magnitudes que pudieran servir de preámbulo para la inclusión de técnicas algebraicas.

T5. Teorías asociadas cuyo propósito fue establecer la significación y el uso del modelo aritmético.

Los autores recuperaron y analizaron los casos de José y de Luis, a partir de un entramado de categorías de la TAD y de la TSD. En relación al momento del primer encuentro ambos estudiantes tienen dificultades para instalar el medio. José hace una "devolución parcial" porque devuelve la tarea, pero no se preocupa por realizar acciones que permiten al alumno recuperar el significado de la tarea antes de intentar solucionarla. Luis da la instrucción de iniciar la actividad, y es hasta el momento en que los niños comienzan a establecer relaciones, que interviene haciendo preguntas para que comprendan la actividad. Los autores llaman a este uso de las preguntas "devolución socrática".

En el momento de la exploración del tipo de tareas $T$ y de la técnica $\tau$ relativa a ellas, José ofrece información parcial, ayudas sobre la manera como pueden resolver y brinda técnicas posibles, y Luis hace una evocación de problemas resueltos con antelación.

En el momento de la constitución del entorno tecnológico-teórico relativo a $\tau$, José recurre al planteamiento de preguntas, una especie de mayéutica. Las preguntas se utilizan en ocasiones para orientar la comprensión de la tarea y en otras para orientar el proceso de identificación de la técnica. Luis recurre a la función explicativa del entorno tecnológico para que los niños entiendan el efecto de una técnica en el proceso de resolución del problema.

Los autores muestran que en ambos casos, el momento de la institucionalización, que tiene por objeto precisar la $\mathrm{OM}$, es complejo y variado. José explica y justifica la técnica como parte del proceso de institucionalización. Luis no puntualiza el concepto de proporcionalidad, pero concluye con los significados construidos por el grupo. Por otro lado, no explica un concepto matemático, sino da cuenta de las relaciones entre las magnitudes implicadas que fueron previamente identificadas por los niños.

En ninguno de los casos hay evaluación de los aprendizajes, aspecto que no fue contemplado en la planeación. 


\section{A MANERA DE CIERRE}

Por su claridad y sencillez en la exposición, este libro puede ser de gran ayuda a los investigadores, docentes y estudiantes que se inicien en la Teoría Antropológica de lo Didáctico y en la didáctica de la proporcionalidad. También brinda un ejemplo de lo que puede ser un REI como dispositivo de estudio en matemáticas.

Desde el punto de vista de la metodología de investigación, el trabajo incorpora un planteamiento novedoso a las perspectivas de las ingenierías didácticas de desarrollo y de la intervención educativa.

Sin duda el gran aporte de este libro es su contribución a la discusión de la formación inicial de docentes desde la Didáctica de las Matemáticas, en particular desde la TAD. La formación de docentes es pensada en el centro de diversos cruces: la confluencia de Praxeologías matemáticas y Praxeologías didácticas; la confluencia de al menos dos instituciones -la escuela de educación básica y la escuela normal-, la confluencia de diversos roles del estudiante normalista, como aprendices, como profesores analistas, como profesores ingenieros, y como profesor en acto.

Queda abierta una interrogante que plantearon los autores: ¿Y la praxeología de los formadores de formadores?

\section{REFERENCIAS}

Aguayo, L. M. y Soto, M. (2020). Enseñar a enseñar Matemáticas. Un recorrido de estudio de investigación para la formación de profesores. UPN-Taberna libraría editores.

Chevallard, Y. (1991). La transposición didáctica. Del saber sabio al saber enseñado. AIQUE. Chevallard, Y. (1994). Les processus de transposition didactique et leur théorisation. En Arsac, G., Chevallard, Y. y Martinand, A. (1994). La transposition didactique à l'épreuve. La pensée sauvage, pp. 135-180.

Chevallard, Y. (2002). Organiser l'étude. 1. Structures y fonctions. En Dorier, J.L., Artaud, M., Artigue, M.. Berthelot, R. y Floris, R. (Eds.). (2002). Actes de la 11è école de Didactique des Mathématiques. La pensée sauvage.

Alejandra Avalos-Rogel

Domicilio: Lago Tláhuac No. 4 - 212 Col. Anáhuac, 11320 Miguel Hidalgo, Ciudad de México, México.

Teléfono: $\quad 5591956018$ 